\title{
Accuracy of Percutaneous Pedicle Screw Placement after Single-Position versus Dual-Position Insertion for Lateral Interbody Fusion and Pedicle Screw Fixation Using Fluoroscopy
}

\author{
Akihiko Hiyama, Hiroyuki Katoh, Daisuke Sakai, Masato Sato, Masahiro Tanaka, Masahiko Watanabe \\ Department of Orthopaedic Surgery, Tokai University School of Medicine, Isehara, Japan
}

\begin{abstract}
Study Design: Retrospective study.
Purpose: The purpose of this study was to compare the accuracy of percutaneous pedicle screw (PPS) placement between prone and lateral decubitus positions during lateral lumbar interbody fusion (LLIF) and to evaluate the tendency of PPS positioning based on simple computed tomography measurements with patients in the lateral decubitus position.

Overview of Literature: There is insufficient information in the literature regarding the accuracy of inserting a PPS using fluoroscopy in patients in the lateral decubitus position.

Methods: We included 62 patients who underwent combined LLIF surgery and PPS fixation for degenerative lumbar spondylolisthesis with spinal canal stenosis. We compared the patient demographics and the accuracy of fluoroscopy-guided PPS placement between two groups: patients who remained in the lateral decubitus position for the pedicle screw fixation (single-position surgery [SPS] group) and those who were turned to the prone position (dual-position surgery [DPS] group).

Results: There were 40 patients in the DPS group and 22 in the SPS group. Of the 292 PPSs, only 12 were misplaced. In other words, 280/292 screws (95.9\%) were placed correctly in the pedicle's cortical shell (grade 0). PPS insertion did not cause neurological, vascular, or visceral injuries in either group. The breach rates for the DPS and SPS groups were 4.1\% (grade 1, 5 screws; grade 2, 3 screws; grade 3, 0 screw) and 4.1\% (grade 1, 2 screws; grade 2, 2 screws; grade 3, 0 screw), respectively. Although there were no statistically significant differences, the downside PPS had more screw malpositioning than the upside PPS.

Conclusions: We found that PPS insertion with the patient in the decubitus position under fluoroscopic guidance might be as safe and reliable a technique as PPS insertion in the prone position, with a misplacement rate similar to that previously published.
\end{abstract}

Keywords: Lateral lumbar interbody fusion; Single-position surgery; Dual-position surgery; Percutaneous pedicle screw; Accuracy

\section{Introduction}

Lateral lumbar interbody fusion (LLIF) is a minimally invasive surgical technique that allows access to the intervertebral disc space and vertebral bodies using the retroperitoneal transpsoas approach [1,2]. In Japan, posterior

Received Oct 16, 2020; Revised Nov 3, 2020; Accepted Nov 19, 2020

Corresponding author: Akihiko Hiyama

Department of Orthopaedic Surgery, Surgical Science, Tokai University School of Medicine, 143 Shimokasuya, Isehara, Kanagawa 2591193, Japan

Tel: +81-463-93-1121, Fax: +81-463-96-4404, E-mail: a.hiyama@tokai-u.jp 
spinal fusion is recommended after LLIF. In such cases, a percutaneous pedicle screw (PPS) is often employed. PPS placement is gradually being adopted for lumbar and thoracic spine surgery and is performed as a minimally invasive spinal fusion (MIST) approach [3,4]. A previous study showed that single-position surgery (SPS) could reduce the mean surgical time by approximately 34 minutes compared with dual-position surgery (DPS) [5].

In general, inaccurate PPS placement can cause potential postoperative neurological deficits [6-9]. Previous reports have indicated $4.7 \%-15 \%$ rates of inaccurate PPS placement; however, PPS placement is reported to be an effective technique with no difference in perforation rate from the standard open free-hand technique [10-12].

A number of authors have reported accurate PPS placement using computer-assisted orthopedic surgery (CAOS) systems, such as intraoperative computer-assisted navigation systems $[13,14]$. These authors have also reported clinical results similar to those of conventional open procedures. However, CAOS procedures are not employed in all hospitals due to the increased associated costs, longer set-up times, and technical difficulties. In addition, neurosurgeons and their teams need to be well trained before using CAOS and navigation systems and to spend more time preparing for surgery. Moreover, there is insufficient information regarding the accuracy of PPS insertion with patients in the lateral decubitus position. Similar to conventional open surgery, this fluoroscopy-guided procedure has been described only by Blizzard and Thomas [1]. It is therefore important to evaluate the SPS and DPS approaches and determine whether PPS placement can be performed accurately with patients in the decubitus position using fluoroscopy. In this study, we aimed to compare the accuracy of PPS placement between the prone and lateral decubitus positions during LLIF and evaluate the tendency of PPS positioning based on simple computed tomography (CT) measurements with patients in the lateral decubitus position.

\section{Materials and Methods}

\section{Included patients}

The study protocol was reviewed and approved by the Committee on Ethics and the Institutional Review Board of Tokai University School of Medicine, the House Clinical Study Committee, and the Profit Reciprocity Com- mittee (Japan; IRB approval no., 20R-038). Given that the study was retrospective, the requirement for informed consent was waived. We retrospectively reviewed the data from cases that met the following inclusion criteria: patient age $>18$ years, lumbar spinal canal stenosis, having undergone a combined operation (indirect decompression) using LLIF with degenerative lumbar spondylolisthesis at a single center between January 2016 and July 2020. The SPS was started in January 2019. The surgical method (SPS or DPS) was determined at the surgeon's discretion, given that there are no detailed inclusion criteria at our hospital for selecting between SPS and DPS.

All patients were diagnosed based on a detailed review of their history, a neurological and radiographic examination, myelograms, CT scans after myelography, and/or magnetic resonance imaging. The location of the stenosis was recorded by the operating neurosurgeon based on an evaluation of the preoperative imaging studies. The coronal Cobb angle of the lumbar vertebral body between the L1 vertebra's upper edges and L5 vertebra's lower edges was evaluated. The mean Cobb angle was $7.1^{\circ} \pm 6.4^{\circ}$, and those patients with an angle $>30^{\circ}$ were excluded.

All patients underwent a postoperative CT scan, and the images were entered into a database for review. The exclusion criteria included undergoing combined procedures, including direct posterior decompression and posterior lumbar fusion, and not undergoing a postoperative CT evaluation.

\section{The operative technique (extreme lateral interbody fusion and percutaneous pedicle screw fixation)}

All patients underwent minimally invasive LLIF surgery utilizing the extreme lateral interbody fusion (XLIF) and PPS insertion technique, as previously described [2,5]. For this study, the upside was on the left side for all patients. Following this procedure, the patients in the DPS group were turned to the prone position and then reprepared and redraped. We then performed bilateral PPS surgery with the patients in the prone position. Patients in the SPS group remained in the lateral decubitus position for PPS fixation (Fig. 1). The C-arm (see below) required finetuning in the craniocaudal direction to align the anterior endplate of the vertebra with the posterior endplate that was turned around to allow visibility of the pedicle symmetrically toward the vertebral rotation angle on an anteroposterior view. 

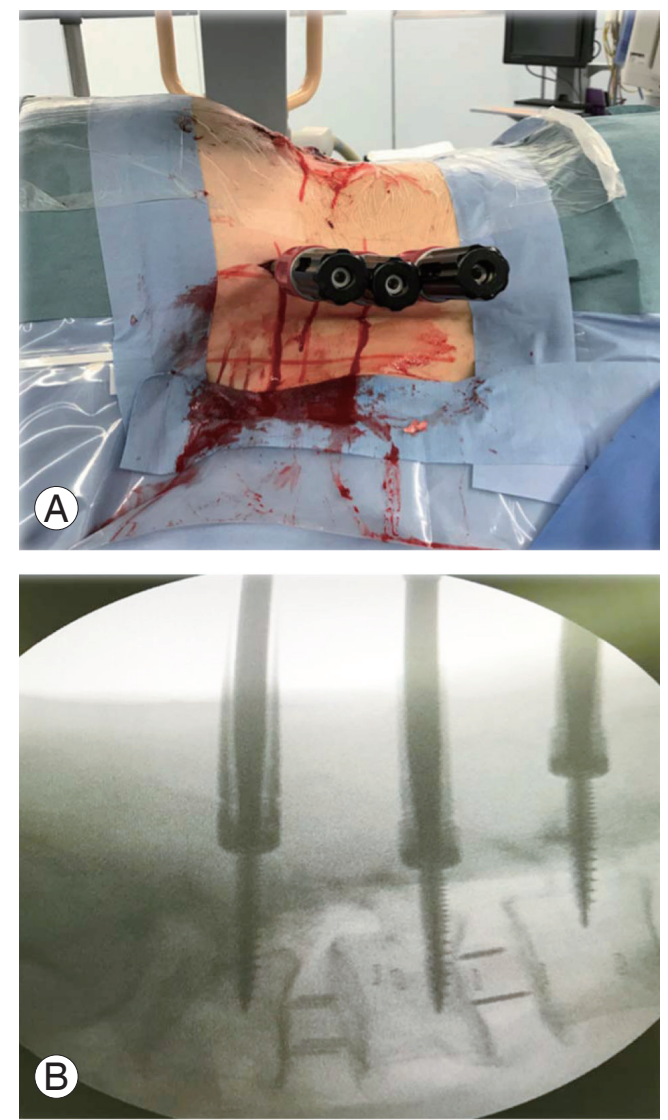

Fig. 1. (A, B) Intraoperative photograph of percutaneous pedicle screw insertion in the lateral decubitus position.

For the DPS and SPS groups, most of the PPS procedures employed the guidewire-less VIPER PRIME system (DePuy Synthes Spine, Raynham, MA, USA) ( $\mathrm{n}=35$, $56.5 \%)$. In particular, we employed the VIPER PRIME system for 18 of the 22 patients in the SPS group (81.8\%). An X-ray image in the anteroposterior view was taken to mark the pedicles' lateral radiographic borders for screw placement. Using a lateral view, the center of each pedicle was identified and marked. A small incision $2-3 \mathrm{~cm}$ lateral to the radiographic borders of each pedicle was made for percutaneous exposure, and the stylets were then docked at the junction of the transverse process and the superior articular process. The stylets were then inserted with a hammer to hold the spot within the pedicles. After the stylets had been inserted into the pedicle's inner rim, an X-ray image in the anteroposterior view was taken to confirm that the posterior body wall had been reached. At that point, the $\mathrm{C}$-arm was brought to a lateral position to maximize the working space for screw placement. After all screws had been inserted, a rod was passed percutane-

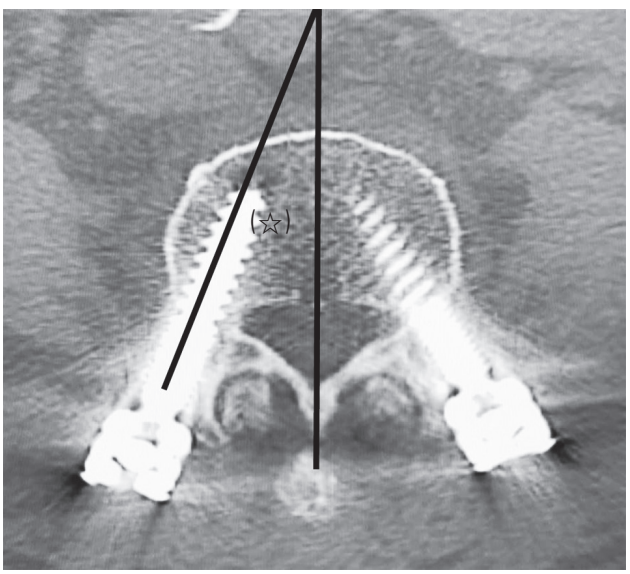

Fig. 2. The insertion angle (访) was measured by subtracting the angle between the line passing through the center of the percutaneous pedicle screw after surgery and using axial computed tomography scans.

ously and secured to the screw heads using setscrews.

\section{Postoperative computed tomography assessment}

Postoperative serial CT scans (in 2.0-mm increments) were obtained for all patients to assess the implant positions. The accuracy of the PPS placement in the medial/ lateral pedicle walls was evaluated with axial CT scans, whereas the superior/inferior pedicle wall screw location was examined using reconstructed sagittal or coronal CT scans. Assessments were performed by an independent spinal neurosurgeon and by the co-authors. PPS misplacement was defined as a breach of the pedicular wall and graded in 2-mm steps (grade 0 , no breach; grade 1, breach $<2 \mathrm{~mm}$; grade 2 , breach $2-4 \mathrm{~mm}$; grade 3 , complications such as pedicle fracture, anterior breach with neurovascular compromise, and lateral/medial breach with neurological sequelae), as described by Raley and Mobbs [15]. We calculated the perforation rate as follows: inaccurate PPS placement (number of screws, grades 1,2, and 3)/ total screws.

We also measured the insertion angle by subtracting the angle between the line passing through the center of the pedicle screw after surgery, using axial CT scan (Fig. 2).

\section{Statistical analysis}

We performed the statistical analyses using IBM SPSS Statistics ver. 20.0 (IBM Corp., Armonk, NY, USA), and all values are expressed as the mean \pm standard deviation. 
Table 1. Comparison of two groups

\begin{tabular}{lccc}
\hline Characteristic & DPS group & SPS group & $p$-value \\
\hline No. of patients & 40 & 22 & \\
\hline Age (yr) & $69.0 \pm 11.7$ & $72.8 \pm 9.2$ & 0.145 \\
\hline Sex (male:female) & $25: 15$ & $12: 10$ & 0.545 \\
\hline Fusion segments & $1.5 \pm 0.8$ & $1.3 \pm 0.5$ & 0.234 \\
\hline One-level & 24 & 16 & \\
\hline Two-levels & 12 & 6 & \\
\hline Three-levels & 3 & 0 & \\
\hline Four-levels & 1 & 0 & \\
\hline Fusion spine levels & 61 & 28 & \\
\hline L1-2 (no. of patients) & 2 & 0 & \\
\hline L2-3 (no. of patients) & 12 & 0 & \\
\hline L3-4 (no. of patients) & 22 & 9 & \\
\hline L4-5 (no. of patients) & 25 & 19 & 0.251 \\
\hline Operative time (min) & $125.9 \pm 37.2$ & $90.2 \pm 26.0$ & $<0.001^{* * *}$ \\
\hline Blood loss (mL) & $72.5 \pm 82.3$ & $52.9 \pm 60.3$ & 0.395 \\
\hline Length of stay (day) & $16.1 \pm 3.7$ & $15.2 \pm 4.8$ & \\
\hline
\end{tabular}

Values are presented as number or mean \pm standard deviation. DPS, dual-position surgery; SPS, single-position surgery.

${ }^{* * *} p<0.001$ (statistically significant).

We assessed the univariate differences between the DPS and SPS groups using independent-sample Student $t$-tests or the Mann-Whitney nonparametric $U$-test for non-normally distributed data. We employed chi-square tests and Fisher's exact test (two-tailed) to determine the significant differences in PPS malpositioning between the two groups. For all statistical analyses, we set the type 1 error at $5 \%$ and considered $p<0.05$ to be statistically significant.

\section{Results}

The study included 62 patients (mean age, $70.3 \pm 11.0$ years; 37 men, 25 women), 40 in the DPS group and 22 in the SPS group. Table 1 lists the patient characteristics and operative details. The most common level treated in both groups was L4-5. Fusion was performed at L1-2 for two patients, at L2-3 for 12 , at L3 -4 for 31 , and at L4 -5 for 44. The estimated mean blood loss and length of stay did not differ between the two groups; however, the operative time was 35 minutes shorter for the SPS group $(90.2 \pm 26.0$ minutes) than for the DPS group (125.9 \pm 37.2 minutes, $p<0.001$ ) (Table 1).

A total of 292 screws were inserted into 62 patients
Table 2. Summary of accuracy data between two groups

\begin{tabular}{ccccc}
\hline Characteristic & DPS group & SPS group & All & $p$-value \\
\hline No. of total screws & 195 & 97 & 292 & \\
\hline Grade 0 & 187 & 93 & 280 & \\
Grade 1 & 5 & 2 & 7 & \\
Grade 2 & 3 & 2 & 5 & \\
Grade 3 & 0 & 0 & 0 & \\
Inaccuracy (\%) & 4.1 & 4.1 & 4.1 & 0.993 \\
\hline No. of reoperation & 0 & 0 & 0 & \\
\hline
\end{tabular}

DPS, dual-position surgery; SPS, single-position surgery.

Table 3. Summary of accuracy data in the fusion spine levels

\begin{tabular}{lccrccc} 
Level & $\begin{array}{c}\text { No. of } \\
\text { screws (\%) }\end{array}$ & $\begin{array}{c}\text { Misplaced } \\
\text { screws (\%) }\end{array}$ & Grade 0 & Grade 1 & Grade 2 & Grade 3 \\
L1 & $4(1.4)$ & $1(8.3)$ & 3 & 1 & 0 & 0 \\
L2 & $24(8.2)$ & $2(16.7)$ & 22 & 0 & 2 & 0 \\
L3 & $74(25.3)$ & $2(16.7)$ & 72 & 2 & 0 & 0 \\
L4 & $107(36.6)$ & $7(58.3)$ & 100 & 4 & 3 & 0 \\
L5 & $83(28.4)$ & 0 & 83 & 0 & 0 & 0 \\
Total & 292 & 12 & 280 & 7 & 5 & 0 \\
\hline
\end{tabular}

Table 4. Summary of accuracy data in the SPS group

\begin{tabular}{lcccccc} 
& \multicolumn{2}{c}{ PPS } & & \multicolumn{2}{c}{ Misplaced screws } & \\
\cline { 2 - 3 } & Upside & Downside & & Upside & Downside & \\
L3 & 9 & 8 & 0 & 0 & - \\
L4 & 22 & 21 & 1 & 3 & 0.272 \\
L5 & 19 & 18 & 0 & 0 & - \\
Total & 50 & 47 & 1 & 3 & 0.278 \\
\hline
\end{tabular}

SPS, single-position surgery; PPS, percutaneous pedicle screw.

alThis $p$-value is the result of a comparison difference between upside PPSs and downside PPSs.

from L1 to L5. The breach rates for the DPS and SPS groups were $4.1 \%(8 / 195)$ and $4.1 \%$ (4/97), respectively, with no statistically significant difference between them ( $p=0.993)$. In terms of misplacement in the DPS group, 187 screws were grade 0,5 screws were grade 1,3 screws were grade 2 , and no screws were grade 3 . In the SPS group, 93 screws were grade 0,2 screws were grade 1 , and 2 screws were grade 2 (Table 2). There were no anterior vertebral perforations for any of the screws, and there were no neurological, vascular, or visceral injuries. Table 3 lists the levels of inaccuracy. We then examined the PPS 


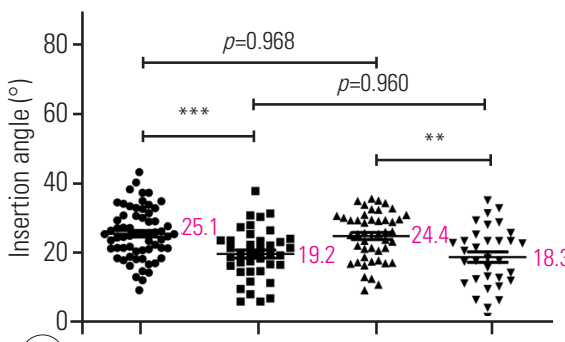

(A) DP-PPS (L4) SP-PPS (L4) DP-PPS (L5) SP-PPS (L5)
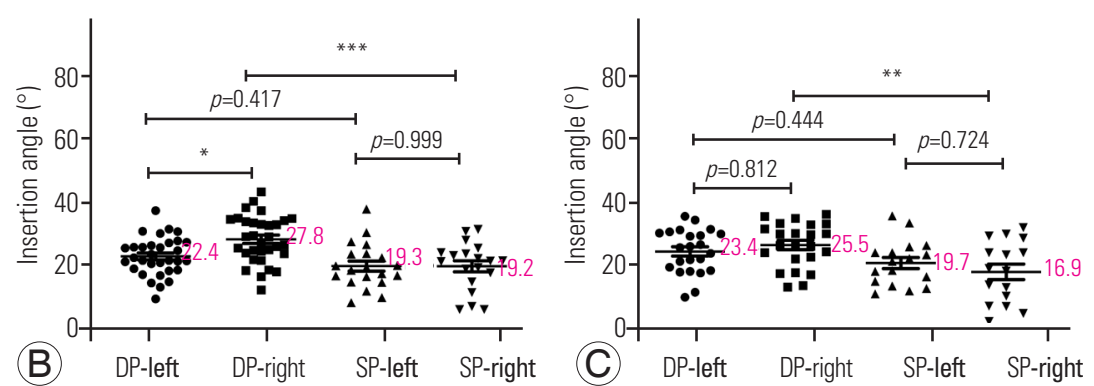

Fig. 3. (A) Comparison of the insertion angle of percutaneous pedicle screws in $L 4$ and $L 5(n=190)$ after surgery between the dual-position surgery (DPS) and singleposition surgery (SPS) groups. (B) Comparison between the DPS and SPS groups in L4. (C) Comparison between the DPS and SPS groups in L5. DP-PPS, dual-position percutaneous pedicle screw; SP-PPS, single-position percutaneous pedicle screw. ${ }^{*} p<0.05$, ${ }^{* *} p<0.01$, and ${ }^{* * *} p<0.001$ between groups.

accuracy in the SPS group given the possible difference in difficulty when inserting the upside PPSs and downside PPSs. There was no statistically significant difference between the upside PPSs and the downside PPSs $(p=0.278)$ (Table 4). The insertion angle at L4 and L5 showed a significant difference between the DPS and SPS groups (L4, $p<0.001$; L5, $p<0.01$ ). When examined in L4 and L5, the insertion angle was significantly smaller for the SPS approach, which involves a downside (right side) PPS, compared with the DPS approach (L4, $p<0.001 ; \mathrm{L} 5, p<0.01)$ (Fig. 3). All pedicle breaches were directed laterally (Fig. 4). In this series, there were no complications related to the PPS insertion, and there were no cases in which reoperation was performed due to PPS deviation.

\section{Discussion}

Pedicle screw-based posterior stabilization systems were introduced in 1984 [16]. Since then, several studies have described percutaneous techniques for posterior lumbar fusion that aimed to prevent iatrogenic injury to the less extensive soft tissue [17-19]. However, the procedures involve longer fluoroscopy times and more implant-related complications because the pedicles are treated blindly or not visualized. In particular, PPS malalignment can cause neurovascular injuries and pedicle fractures, as well as pseudoarthrosis due to instability. With this background in mind, several studies have reported on the differences in PPS placement accuracy between open procedures and MIST using conventional fluoroscopy to determine that procedure has better accuracy in PPS placement [20].

Previous reports on the accuracy of PPS placement have shown that the most widely used postoperative imaging technique is CT $[6,15,21]$. However, there is no gold standard for identifying PPS positions based on this imaging
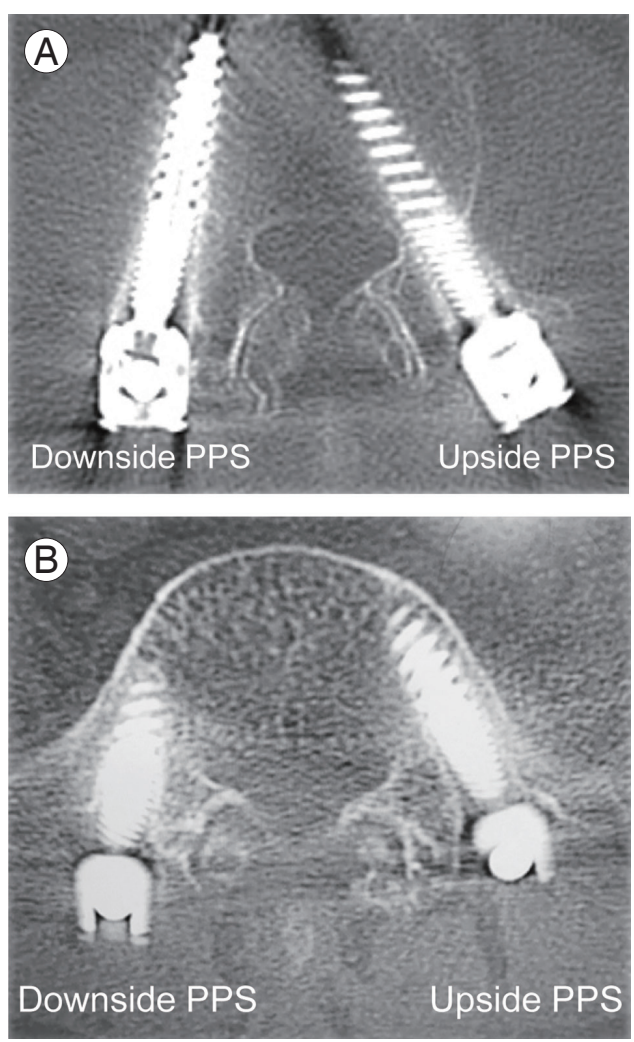

Fig. 4. A 73-year-old female patient with degenerative spondylolisthesis underwent L4-5 XLIF+SP-PPS. (A) Immediate postoperative computed tomography scans showed a lateral misplacement of the L4 PPS on the downside (grade 2). (B) PPS insertion at L5 posed no problem. PPS, percutaneous pedicle screw; XLIF, extreme lateral interbody fusion; SP-PPS, single-position percutaneous pedicle screw.

modality. Penetration of up to $2 \mathrm{~mm}$ into the epidural space is considered clinically safe in most cases. When CT is employed for PPS placement, there can be an error of 1-2 mm caused by metal artifacts [20]. We therefore evaluated the accuracy of PPS placement using a 2-mm incremental grading system based on CT [15].

Although the effectiveness of indirect decompression 
using LLIF has been reported [22,23], many current procedures require the patient to be repositioned to the prone position after LLIF for PPS fixation. This repositioning requires rearranging the operating room, which increases the operative time and costs due to the extra use of materials. Thus, if surgery could be completed in the lateral decubitus position, the surgical invasion degree would be minimized.

The current PPS insertion technique using a guidewire, first described by Holly and Foley [24], has become the fundamental technique for MIST. Several instruments, such as a needle, guidewire, tap, and screwdriver, were required to insert a conventional PPS. Inserting the PPS in the lateral decubitus position is therefore complicated, and the working space is reduced. Given this information, there have been numerous reports on procedures with patients in the lateral decubitus position using CAOS. By including intraoperative $\mathrm{CT}$ and navigation, CAOS can certainly be considered to provide safer PPS placement [25]; however, certain navigation drawbacks should be considered, such as longer operative times, steeper learning curves, and higher costs. Moreover, this is not a technique that can be performed at all facilities and by all spinal neurosurgeons, thus the importance of investigating the accuracy of PPS insertion in the lateral decubitus position using fluoroscopy. We therefore evaluated the screw placement accuracy of PPS between the SPS and DPS groups. The results showed that the DPS approach was inaccurate in $4.1 \%$ of the 195 screws, while the SPS approach was inaccurate in $4.1 \%$ of the 97 screws. The downside PPSs in the SPS group tended to be misplaced; however, this trend was not statistically significant. Generally, the entry point of the percutaneous lumbar PPS is more lateral than that of the open procedure, and its pathway is steep on the axial plane. Ebraheim et al. [26] analyzed the directions of screw misplacements and showed significantly more lateral than medial pedicle violations at the lumbar levels. The authors also showed that half of these misplacements could be attributed to incorrect insertion angles and suggested that these incorrect angles could be explained by an increasing medial inclination of the pedicle in the transverse plane when descending from L1 $\left(17^{\circ}-25^{\circ}\right)$ to L5 $\left(26^{\circ}-40^{\circ}\right)$ [26]. We therefore compared the PPS insertion angle between the SPS and DPS groups. The insertion angle at L4 and L5 showed a clear difference between the SPS and DPS groups, which appeared to be due to the interference of the bed frame when inserting

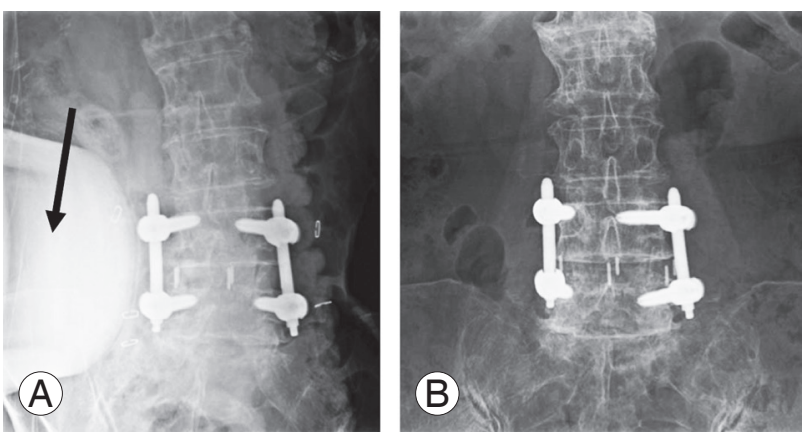

Fig. 5. This figure is the same case as in Fig. 4. (A) Intraoperative radiograph evaluation. (B) Postoperative radiograph evaluation. Immediately after surgery, the radiograph did not seem to cause any problems with percutaneous pedicle screw (PPS) insertion. However, because we put a pillow (arrow) under the patient's body, the patient's body was rotated slightly, so we made the wrong decision during surgery and did not notice the PPS's lateral displacement.

the PPS in the lateral decubitus position. Spinal surgeons need to be careful with the insertion angle when patients are in the lateral decubitus position. Care should also be taken when bending the patient's body to maximize the spine's visibility, as the lumbar spine can undergo rotation and scoliosis (Fig. 5). Therefore, we now take care not to bend the patient's body too much with the SPS-PPS technique.

However, this study suggested no difference in the PPS accuracy rate despite the difference in the PPS insertion angle and the fact that the insertion angle does not directly lead to PPS misplacement. The findings also suggested that the effect of body position on the accuracy of PPS insertion was small.

This study had a number of limitations, including its retrospective design using data based on medical records and the lack of complete clinical information, which might have caused uncertainty in the results. Other limitations were the small number of patients and the difference in the diameters and lengths of the inserted PPSs. Lastly, this study did not include other radiology results or clinical data such as bone fusion rates, screw failures, and information on the patients' postoperative quality of life.

\section{Conclusions}

A total of 280 of the 292 PPSs (95.9\%) were placed correctly in the pedicle's cortical shell, and the breach rates in the DPS and SPS groups were both $4.1 \%$. There is no doubt that further research into screw precision is warranted. This study has shown that PPS insertion under fluoroscopy with the patient in the decubitus position 
might be a similarly safe and reliable technique compared with PPS insertion in the prone position, with a misplacement rate similar to that published in the literature. The SPS approach for the indirect decompression might represent an attractive option for treating degenerative lumbar spondylolisthesis due to its short surgical time and no difference in screw accuracy when compared with DPS.

\section{Conflict of Interest}

No potential conflict of interest relevant to this article was reported.

\section{Author Contributions}

All authors have read and approved the manuscript. AH conceived and designed the study and interpreted the results; HK analyzed the data and interpreted the results; DS acquired the data and interpreted the results; MS acquired the data and interpreted the results; MT analyzed and acquired the data; and MW conceived and designed the study and interpreted the results.

\section{References}

1. Blizzard DJ, Thomas JA. MIS single-position lateral and oblique lateral lumbar interbody fusion and bilateral pedicle screw fixation: feasibility and perioperative results. Spine (Phila Pa 1976) 2018;43:440-6.

2. Ozgur BM, Aryan HE, Pimenta L, Taylor WR. Extreme lateral interbody fusion (XLIF): a novel surgical technique for anterior lumbar interbody fusion. Spine J 2006;6:435-43.

3. Smith BW, Joseph JR, Kirsch M, Strasser MO, Smith J, Park P. Minimally invasive guidewireless, navigated pedicle screw placement: a technical report and case series. Neurosurg Focus 2017;43:E9.

4. Wang H, Zhou Y, Li C, Liu J, Xiang L. Comparison of open versus percutaneous pedicle screw fixation using the sextant system in the treatment of traumatic thoracolumbar fractures. Clin Spine Surg 2017;30:E239-46.

5. Hiyama A, Sakai D, Sato M, Watanabe M. The analysis of percutaneous pedicle screw technique with guide wire-less in lateral decubitus position following extreme lateral interbody fusion. J Orthop Surg Res 2019;14:304.
6. Ravi B, Zahrai A, Rampersaud R. Clinical accuracy of computer-assisted two-dimensional fluoroscopy for the percutaneous placement of lumbosacral pedicle screws. Spine (Phila Pa 1976) 2011;36:84-91.

7. Kim MC, Chung HT, Cho JL, Kim DJ, Chung NS. Factors affecting the accurate placement of percutaneous pedicle screws during minimally invasive transforaminal lumbar interbody fusion. Eur Spine J 2011;20:1635-43.

8. Wood MJ, Mannion RJ. Improving accuracy and reducing radiation exposure in minimally invasive lumbar interbody fusion. J Neurosurg Spine 2010;12:533-9.

9. Yoshida G, Sato K, Kanemura T, Iwase T, Togawa D, Matsuyama Y. Accuracy of percutaneous lumbosacral pedicle screw placement using the oblique fluoroscopic view based on computed tomography evaluations. Asian Spine J 2016;10:630-8.

10. Heintel TM, Berglehner A, Meffert R. Accuracy of percutaneous pedicle screws for thoracic and lumbar spine fractures: a prospective trial. Eur Spine J 2013;22:495-502.

11. Chiu CK, Chan CYW, Kwan MK. The accuracy and safety of fluoroscopic-guided percutaneous pedicle screws in the thoracic and lumbosacral spine in the Asian population: a CT scan analysis of 1002 screws. J Orthop Surg (Hong Kong) 2017;25:2309499017713938.

12. Chapman TM, Blizzard DJ, Brown CR. CT accuracy of percutaneous versus open pedicle screw techniques: a series of 1609 screws. Eur Spine J 2016;25:1781-6.

13. Fomekong E, Safi SE, Raftopoulos C. Spine navigation based on 3-dimensional robotic fluoroscopy for accurate percutaneous pedicle screw placement: a prospective study of 66 consecutive cases. World Neurosurg 2017;108:76-83.

14. Ohba T, Ebata S, Fujita K, Sato H, Haro H. Percutaneous pedicle screw placements: accuracy and rates of cranial facet joint violation using conventional fluoroscopy compared with intraoperative threedimensional computed tomography computer navigation. Eur Spine J 2016;25:1775-80.

15. Raley DA, Mobbs RJ. Retrospective computed tomography scan analysis of percutaneously inserted pedicle screws for posterior transpedicular stabilization of the thoracic and lumbar spine: accu- 
racy and complication rates. Spine (Phila $\mathrm{Pa} 1976$ ) 2012;37:1092-100.

16. Magerl FP. Stabilization of the lower thoracic and lumbar spine with external skeletal fixation. Clin Orthop Relat Res 1984;(189):125-41.

17. Harris EB, Massey P, Lawrence J, Rihn J, Vaccaro A, Anderson DG. Percutaneous techniques for minimally invasive posterior lumbar fusion. Neurosurg Focus 2008;25:E12.

18. Ringel F, Stoffel M, Stuer C, Meyer B. Minimally invasive transmuscular pedicle screw fixation of the thoracic and lumbar spine. Neurosurgery 2006;59(4 Suppl 2):ONS361-6.

19. Foley KT, Gupta SK. Percutaneous pedicle screw fixation of the lumbar spine: preliminary clinical results. J Neurosurg 2002;97(1 Suppl):7-12.

20. Aoude AA, Fortin M, Figueiredo R, Jarzem P, Ouellet J, Weber MH. Methods to determine pedicle screw placement accuracy in spine surgery: a systematic review. Eur Spine J 2015;24:990-1004.

21. Costa F, Cardia A, Ortolina A, Fabio G, Zerbi A, Fornari M. Spinal navigation: standard preoperative versus intraoperative computed tomography data set acquisition for computer-guidance system: radiological and clinical study in 100 consecutive patients. Spine (Phila Pa 1976) 2011;36:2094-8.
22. Campbell PG, Nunley PD, Cavanaugh D, et al. Shortterm outcomes of lateral lumbar interbody fusion without decompression for the treatment of symptomatic degenerative spondylolisthesis at L4-5. Neurosurg Focus 2018;44:E6.

23. Nakashima H, Kanemura T, Satake K, et al. Indirect decompression on MRI chronologically progresses after immediate postlateral lumbar interbody fusion: the results from a minimum of 2 years follow-up. Spine (Phila Pa 1976) 2019;44:E1411-8.

24. Holly LT, Foley KT. Three-dimensional fluoroscopyguided percutaneous thoracolumbar pedicle screw placement: technical note. J Neurosurg 2003;99(3 Suppl):324-9.

25. Sellin JN, Mayer RR, Hoffman M, Ropper AE. Simultaneous lateral interbody fusion and pedicle screws (SLIPS) with CT-guided navigation. Clin Neurol Neurosurg 2018;175:91-7.

26. Ebraheim NA, Rollins JR Jr, Xu R, Yeasting RA. Projection of the lumbar pedicle and its morphometric analysis. Spine (Phila Pa 1976) 1996;21:1296-300. 